O. Echt and K. Sattler, unpublished results. We appreciate these authors informing us of their work prior to publication.

${ }^{10}$ C. L. Briant and J. J. Burton, J. Chem. Phys. $\underline{63}$, 2045 (1975).

${ }^{11}$ There are two more experimental points by T. Pierce, P. M. Sherman, and D. D. McBride, Astro- naut. Acta 16, 1 (1971), that have a nucleation onset at much higher pressure for $T \simeq 55^{\circ} \mathrm{K}$ than the results of Refs. 1 and 2 by Stein and Wu, Wegener, and Stein (see Ref. 2). We have not plotted these points in our Fig. 2 in order not to introduce dispersion in the data, but we stress that more data are necessary at high $T$ to clear up the situation.

\title{
Theory of Spin Relaxation by Magnetic Dipole Interaction in a Fermi Liquid: Application to ${ }^{3} \mathrm{He}$
}

\author{
D. Vollhardt and P. Wölfle \\ Max-Planck-Institut für Physik und Astrophysik, D-8000 München, West Germany, and Physik-Department \\ der Technischen Universität München, D-8046 Garching, West Germany
}

(Received 27 April 1981)

\begin{abstract}
We consider a system of fermions interacting via both strong spin-conserving and weak (spin-nonconserving) magnetic dipole forces. An expression for the spin relaxation rate $1 / T_{1}$ in terms of the incoherent dynamic structure factor $S_{I}(q, \omega)$ is derived by treating the dipole interaction as a perturbation. We have calculated $T_{1}$ for normal liquid ${ }^{3} \mathrm{He}$ at all temperatures using the polarization-potential approach of Aldrich and Pines as well as the measured spin-diffusion coefficient and find good agreement with available data.
\end{abstract}

PACS numbers: $67.50 . \mathrm{Dg}, 76.60 . \mathrm{Es}$

Recently the dynamics of Fermi liquids on the atomic scale has attracted new interest due to both the first neutron scattering data for liquid ${ }^{3} \mathrm{He}$ (Ref. 1) and their theoretical interpretation in terms of the so-called polarization-potential theory. ${ }^{2}$ Since neutron scattering experiments are difficult to perform, one may consider whether there are any other physical quantities that can be employed for probing the dynamics of the liquid at large frequency $\omega$ and/or wave vector $\vec{q}$. In this Letter, we want to show that one such quantity is the spin-relaxation time $T_{1}$, which samples the spin-density excitation spectrum of the liquid for wave vectors up to twice the Fermi wave vector $k_{\mathrm{F}}$.

A nonequilibrium homogeneous spin polarization $\langle S\rangle$ of a Fermi liquid may relax in the bulk because of spin-orbit interactions such as the dipole interaction of the magnetic moments associated with the fermion spins. In the event that the dipole energy is small in comparison with the characteristic energies of the system (which is the case in ${ }^{3} \mathrm{He}$ ), the time scales of the relaxation of the total spin and the motion of the other degrees of freedom (single-particle excitations, collective modes) are well separated and the spin-relaxa- tion rate may be calculated in perturbation theory with respect to the dipole interaction. The elementary spin-relaxation process then consists in the decay of a homogeneous $(\vec{q}=0)$ spin fluctuation into two spin modes with momenta $\pm \vec{q}$ via the spin-orbit force, a process by which spin angular momentum is transformed into orbital angular momentum.

We consider a system of fermions interacting via strong spin-conserving forces and the weak dipole-dipole forces associated with the spin magnetic moments, as described by the Hamiltonian

$$
H_{D}=-\frac{2}{3} \pi \gamma^{2} \sum_{\overrightarrow{\mathrm{q}}}\left[\overrightarrow{\mathrm{S}}_{\overrightarrow{\mathrm{q}}} \cdot \overrightarrow{\mathrm{S}}_{-\overrightarrow{\mathrm{q}}}-3\left(\hat{\boldsymbol{q}} \cdot \overrightarrow{\mathrm{S}}_{\overrightarrow{\mathrm{q}}}\right)\left(\hat{q} \cdot \overrightarrow{\mathrm{S}}_{-\overrightarrow{\mathrm{q}}}\right)\right] \text {. }
$$

Here $\vec{S}_{\vec{q}}$ is the Fourier component of the spin-density operator with wave vector $\vec{q}, \gamma$ is the gyromagnetic ratio, and $\hat{q}=\vec{q} /|\vec{q}|$. The last term in the $\overrightarrow{\mathrm{q}}$ sum in $H_{D}$ clearly breaks spin rotation invariance. It is this interaction that governs the relaxation of the total spin operator $\overrightarrow{\mathrm{S}}=\overrightarrow{\mathrm{S}} \overrightarrow{\mathrm{q}}=0$ :

$$
\dot{\overrightarrow{\mathrm{S}}}=2 \pi \gamma^{2} \hbar^{-1} \sum_{\overrightarrow{\mathrm{q}}}\left[\left(\hat{q} \times \overrightarrow{\mathrm{S}}_{\overrightarrow{\mathrm{q}}}\right)(\hat{q} \cdot \overrightarrow{\mathrm{S}}-\overrightarrow{\mathrm{q}})+\text { H.c. }\right] \text {. }
$$

Our aim is to express the spin-relaxation rate $1 / T_{1}$ in terms of the spin-density response function $\chi(z)$ defined by

$$
\chi(\omega+i 0)=\left(i / \hbar^{2}\right) \int_{0}^{\infty} d t e^{i \omega t}\left\langle\left[S^{z}(t), S^{z}(0)\right]_{-}\right\rangle \equiv\{M(\omega+i 0) /[\omega+M(\omega+i 0)]\} \chi(0),
$$


where in the last equality we have defined a spin-relaxation function $M .^{3}$ For $\omega \rightarrow 0, M(\omega+i 0)$ tends to an imaginary constant $i / T_{1}$, where $T_{1}$ is the longitudinal spin-relaxation time $\left(=T_{2}\right.$, for small magnetic fields). $M$ is expected to vary with frequency on a scale given by the thermal energy $k_{\mathrm{B}} T$, the singleparticle level width $h / \tau$, or the Fermi energy $E_{\mathrm{F}}$, all of which are much larger than the dipole energy $E_{D}=\left\langle H_{D}\right\rangle \simeq \gamma^{2} \hbar^{2} n$ ( $n$ is the particle density). Consequently there exists a frequency range where $\omega T_{1}$ $\gg 1$ and $M(\omega+i 0) \simeq\left(1 / T_{1}\right)\left[i+O\left(\hbar \omega / k_{\mathrm{B}} T, \hbar \omega / E_{\mathrm{F}}, \omega \tau\right)\right]$, such that by expanding (2) in $\left(1 / \omega T_{1}\right)$ one obtains ${ }^{3}$

$$
1 / T_{1}=\left.\omega\left[\operatorname{Im} \chi(\omega+i 0) /\left.\chi(0)\right|_{\omega=0}=\left[\chi(0) \hbar^{2} \omega\right]^{-1} \int_{-\infty}^{\infty} d t e^{i \omega t}\left\langle\left[S^{z}(t), S^{z}(0)\right]\right]_{-}\right\rangle\right|_{\omega=0} .
$$

Substituting $\dot{S}^{z}$ from (1), $1 / T_{1}$ is seen to be given by a four-spin correlation function multiplied by the square of the dipole coupling constant. This complicated correlation function may be factorized into two-spin correlation functions if either the interactions in the system are weak in general or if the spin-density excitations are well defined. The latter is true for ${ }^{3} \mathrm{He}$, where in the collisionless regime the spin fluctuations are strongly enhanced by the spin-polarization potential, while in the hydrodynamic regime there exists a spin-diffusion mode. After the factorization $1 / T_{1}$ may be expressed in terms of the absorptive part of the wave-vector dependent dynamic spin susceptibility $\chi^{\prime \prime}(q, \omega)$ as

$$
\frac{1}{T_{1}}=\frac{1}{3 \pi^{2}} \frac{\left(\gamma^{2} \hbar^{2}\right)^{2}}{\chi(0) k_{\mathrm{B}} T} \int d^{3} q \int d \omega\left(\frac{\chi^{\prime \prime}(q, \omega)}{\sinh \left(\hbar \omega / 2 k_{\mathrm{B}} T\right)}\right)^{2} .
$$

Note that $\chi^{\prime \prime}$ is related to the incoherent dynamic structure factor $S_{I}(q, \omega)=\frac{1}{2} \hbar \chi^{\prime \prime}(q, \omega) /[1-\exp (-\hbar$ $\left.\left.\times \omega / k_{\mathrm{B}} T\right)\right]$, which is measured by magnetic neutron scattering. Measurements of $T_{1}$ can therefore serve to assess the quality of approximations of the dynamic structure factor.

We now turn to the evaluation of (4) using approximations for $\chi^{\prime \prime}(q, \omega)$.

(1) In the collisionless regime, for $q>q_{0}$, we approximate $\chi^{\prime \prime}$ by the polarization-potential expression of Aldrich and Pines, ${ }^{2}$

$$
\chi^{\prime \prime}(q, \omega)=\operatorname{Im}\left[\chi_{\mathrm{sc}^{-1}}(q, \omega+i 0)-f_{0}^{a}(q)\right]^{-1},
$$

where $\chi_{s c}(q, \omega+i 0)$ is a screened spin-density response function and $f_{0}{ }^{a}(q)$ is a wave-vector-dependent but temperature-independent polarization parameter, which tends to the spin-antisymmetric $\boldsymbol{l}=0$ Landau parameter $f_{0}{ }^{a}$ in the limit $q \rightarrow 0$. In the region of the $\omega-q$ plane we are interested in, higher $l$ components of the polarization potential such as the backflow term $(l=1)$ are negligible. We approximate $\chi_{s c}(q, \omega)$ by the $T=0$ Lindhard function multiplied by a strength factor $\alpha(q)$ for single-particle-hole excitations [the contribution of multipair excitations to (4) is negligible in the temperature range considered here] and multiplied by a temperature-dependent factor $\chi_{\text {sc }}{ }^{T}(0,0) / \chi_{\text {sc }}{ }^{T=0}(0,0)$ chosen such as to yield the experimentally determined ${ }^{4}$ spin susceptibility $\chi(0,0)=\left[1 / \chi_{\text {sc }}{ }^{T}(0,0)-f_{0}{ }^{a}(0)\right]^{-1}$. The polarization parameter $f_{0}{ }^{a}(q)$ was calculated numerically along the lines of Ref. 2 taking the relative range difference $\delta\left[\delta=1-r_{\uparrow \downarrow} / r_{\uparrow \uparrow}\right.$, where $r_{\uparrow \downarrow}\left(r_{\uparrow \uparrow}\right)$ is the range of the effective repulsive interaction between particles of antiparallel (parallel) spin $^{5}$ ] equal to $\delta=0.011$ for saturated vapor pressure and $\delta=0.0032$ for $P=27$ bars. $^{6}$ The $q$ dependences of $\alpha(q)$ as plotted in Ref. 2 and of $f_{0}{ }^{a}(q)$ are very well approximated for $q \leqslant 2 k_{\mathrm{F}}$ by the following expressions $\left(y \equiv q / 2 k_{\mathrm{F}}\right): \alpha(q)=1-y^{2}\left(0.416+0.172 y^{4}\right)$ and $f_{0}{ }^{a}(q) / f_{0}{ }^{a}(0)=1-y^{4}\left(1.2-0.5 y^{2}\right)$ for $P=0$ and $f_{0}{ }^{a}(q) / f_{0}{ }^{a}(0)=1-y^{2}\left(0.7+0.1 y^{2}\right)$ for $P=27$ bars. The temperature broadening ${ }^{7}$ of the spectral density $\chi_{s c}{ }^{\prime \prime}(q, \omega) / \omega$ as a function of $\omega$, which is neglected in our approximation, is expected to give rise to a small correction only.

Substituting (5) into (4) the contribution to the spin-relaxation rate in the degenerate Fermi-liquid regime is found (in random-phase approximation) to be

$$
\left(\frac{1}{T_{1}}\right)_{\mathrm{RPA}}=\frac{\pi^{5}}{2 \hbar} \frac{c}{\left(1+F_{0}{ }^{a}\right)^{3}}\left(\gamma^{2} \hbar^{2} n\right)^{2} \frac{\left(k_{\mathrm{B}} T\right)^{2}}{E_{\mathrm{F}}{ }^{3}},
$$

where $F_{0}{ }^{a}$ is the Landau parameter $\left[F_{0}{ }^{a}=-0.67\right.$ $(-0.74)$ at $P=0(P=27$ bars $)]$ and $c$ is a dimensionless function of pressure $[c=0.33(0.24)$ at $P=0(P=27$ bars $)]$. The relaxation rate is seen to be $\propto E_{D}^{2}\left(k_{\mathrm{B}} T\right)^{2} / E_{\mathrm{F}}{ }^{3}$, as found previously by Ipatova and Eliashberg. ${ }^{8}$ However, the prefactor is enhanced by $\left(1+F_{0}{ }^{a}\right)^{-3} \simeq 30-60$ due to spin-polarization effects in the nearly ferromagnetic Fermi liquid ${ }^{3} \mathrm{He}$. In order to investigate the factorization approximation involved in deriving (4) from (3) we have evaluated the four-spin correlation function in (3) by means of an exact factorization for the free Fermi gas and found this to be smaller by a factor of 2 compared with (6) putting $F_{0}{ }^{a}=0$. The additional contribution stems from totally incoherent processes which are not enhanced by spin-polarization effects and therefore can be expected to be much smaller than the main contribution (6). It should be mentioned that 
the result of the exact factorization is identical to the one of Ref. 8 if the angular average appearing in their result is actually performed. In Fig. 1 , the result of a numerical evaluation of $\Gamma_{\mathrm{RPA}}$ $=\left(1 / T_{1}\right)_{\text {RPA }}$ with use of parameter values ${ }^{9}$ appropriate for normal liquid ${ }^{3} \mathrm{He}$ at pressures of 0 and 27 bars is shown as a function of temperature. The prefactor of the $T^{2}$ law [ the constant $c$ in (6)] as well as the high $T$ plateau value depend sensitively on the $q$ dependence of $\chi^{\prime \prime}(q, \omega)$ [i.e., $f_{0}{ }^{a}(q)$ and $\alpha(q)$ ]. Taking $f_{0}{ }^{a}(q)=f_{0}{ }^{a}(0)$ and $\alpha(q)=1$ the relaxation rates $\left(1 / T_{1}\right)_{\mathrm{RPA}}$ are always higher (about a factor of 4 at $P=0$ bar and a factor of 2 at $P=27$ bars).

(2) For $q<q_{0} \simeq 1 / l$, where $l$ is a mean free path (and $\omega<1 / \tau$ ), the dynamics of the system is governed by the hydrodynamic equations and hence $\chi^{\prime \prime}(q, \omega)=D q^{2} \omega /\left(\omega^{2}+D^{2} q^{4}\right) \chi(q, \omega=0)$, where $D$ is the spin-diffusion coefficient. We have approximated $\chi(q, \omega=0)$ by the expression for the collisionless regime which should provide a good interpolation formula. The contribution to the spin-relaxation rate is then given by

$$
\left(1 / T_{1}\right)_{\text {diff }}=(4 \pi / 5) \gamma^{4} \hbar^{2}\left[k_{\mathrm{B}} T \chi(0) / D d(P, T)\right] .
$$

Here we have introduced a temperature- and pressure-dependent length $d(P, T)=(3 \pi / 10)\left(f q_{0}\right)^{-1}$, where $f(P, T)$ is a dimensionless function close to unity, so that $d(P, T) \simeq q_{0}^{-1}$. The deviation of $f$ from 1 depends on the $q$ dependence of $\chi(q, \omega$ $=0)$. The cutoff wave vector $q_{0}$ may be determined by requiring the diffusion broadening at $q$ $=q_{0}$ to be equal to the single-particle level broadening, i.e., $D q_{0}{ }^{2}=1 / \tau$. $D$ is related to $\tau$ by the Einstein relation $D=\left[n / 4 m^{*}(T) \chi(0)\right] \tau$, where we have introduced a temperature-dependent effective mass $m^{*}(T)$ which tends to the Fermi-liquid effective mass and to the bare mass, respectively, in the limits $T \ll T_{\mathrm{F}}$ and $T \gg T_{\mathrm{F}}, m^{*}(T)=\left\langle p^{2}\right\rangle /$ $2\langle E\rangle=\frac{1}{2}\left\{\left[p_{\mathrm{F}}{ }^{4}+\left(3 m k_{B} T\right)^{2}\right] /\left[E_{\mathrm{F}}{ }^{2}+\left(3 k_{\mathrm{B}} T / 2\right)^{2}\right]\right\}^{1 / 2}$. From the definition of $q_{0}$ it follows that in the Fermi-liquid regime $d \propto T^{-2}$ while for $T \gtrsim 0.5 \mathrm{~K}$ $d(P, T)$ levels off to essentially a constant but still pressure-dependent length $d_{c}=1.9 \AA\left(d_{c}=1.0\right.$ $\AA)$ at $P=0(P=27$ bars $)$. The temperature dependence of $d(P, T)$ for $T \lesssim 2.5 \mathrm{~K}$ is well approximated by the expression $d(P, T) / d_{c}=\left[1+\left(B T_{\mathrm{F}}{ }^{2} /\right.\right.$ $\left.\left.T^{2}\right)^{\lambda}\right]^{1 / \lambda}$ with $\lambda=0.63$ and $B=1.5 \times 10^{-2}$. At low $T, \Gamma_{\text {diff }}=\left(1 / T_{1}\right)_{\text {diff }}$ therefore varies as $T^{5}$ so that its contribution to the total relaxation rate is negligible. On the other hand, in the classical regime, when $\chi(0)=S(S+1) n /\left(3 k_{\mathrm{B}} T\right)$, our result (7) reduces to the well-known formula of Bloembergen, Purcell, and Pound ${ }^{10}$ (BPP), which success-
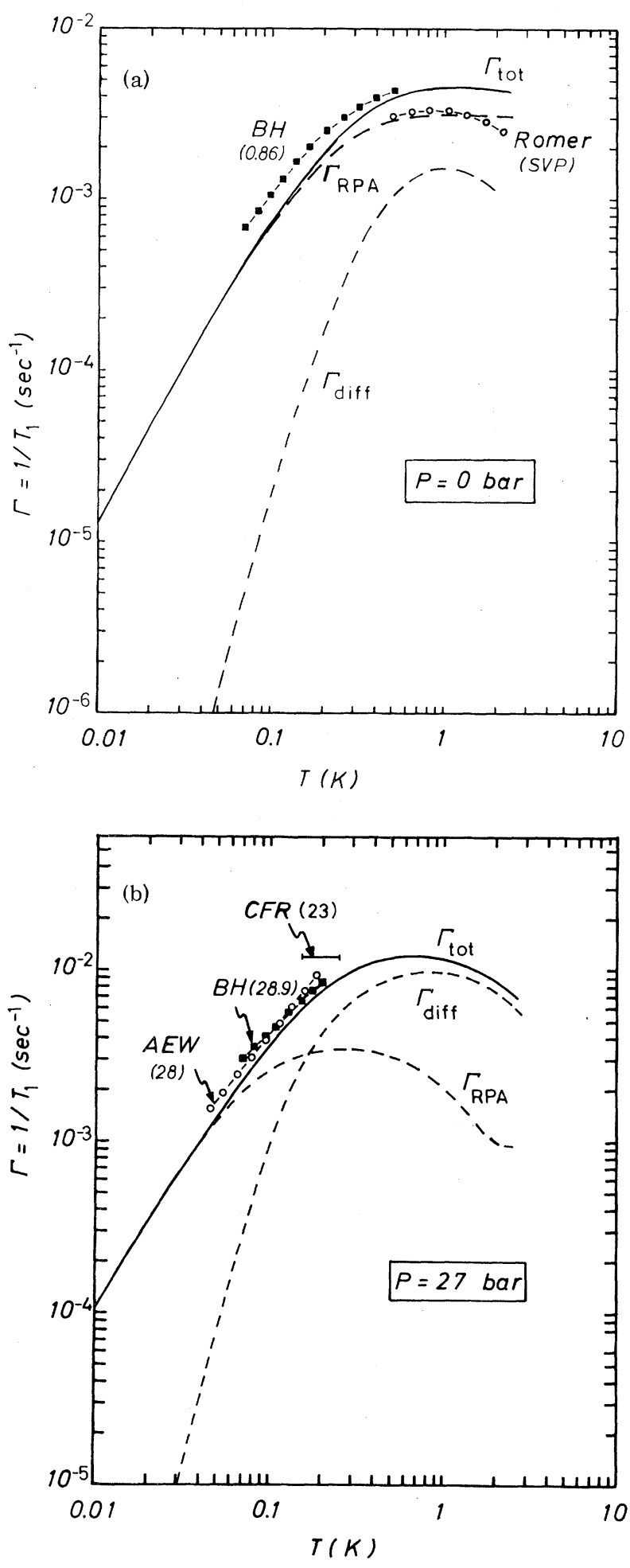

FIG. 1. The two contributions $\Gamma_{\mathrm{d} i f f}$ and $\Gamma_{\mathrm{RPA}}$ (dashed curves) to the total relaxation rate $\Gamma_{\text {tot }}=1 / T_{1}$ (full curve) as functions of temperature $T$ at two pressures: (a) $P=0$ bar and (b) $P=27$ bars (see text). 
fully predicts the relaxation rates of many classical liquids. We observe that $d(P, T)$ is essentially the mean free path, rather than a pressureand temperature-independent length (half of "distance of closest approach," ${ }^{10}$ i.e., about $1 \AA$ for ${ }^{3} \mathrm{He}$ ) and hence (7) is a generalization of the BPP formula to all temperatures $T \lesssim T_{\mathrm{F}}$. For the dense, classical liquid ( $P=27$ bars) both lengths are seen to coincide, however. In Fig. 1, the relaxation rate $\Gamma_{\text {diff }}=\left(1 / T_{1}\right)_{\text {diff }}$ is shown as a function of temperature for two values of pressure ( $P=0$ and 27 bars). The temperature-dependent spin-diffusion constant was taken from Hart and Wheatley ${ }^{11}$ for $P=0$ and from Garwin and Reich $^{12}$ and Wheatley ${ }^{13}$ for $P=27$ bars. Also shown in Fig. 1 is the total spin-relaxation rate $\Gamma_{\mathrm{tot}}=1 / T_{1}=\left(1 / T_{1}\right)_{\mathrm{RPA}}+\left(1 / T_{1}\right)_{\text {diff }}$. In the low-temperature range $(T \lesssim 0.2 \mathrm{~K})$ the contribution from the collisionless regime always dominates the hydrodynamic contribution. At higher temperatures the relative contribution to the relaxation rate of both mechanisms strongly depends on pressure. At low pressure the main contribution is due to particle-hole excitations (liquid ${ }^{3} \mathrm{He}$ at these temperatures and pressures is rather like a dense gas), while at the higher pressure the hydrodynamic contribution takes over at $T \gtrsim 0.5 \mathrm{~K}$, i.e., the relaxation rate is then fairly well described by merely our generalized BPP formula for the dense classical liquid.

We have collected the available experimental data for the relaxation time $T_{1}$ in Fig. 1 (numbers in parentheses indicate experimental pressures measured in bars): In Fig. 1(a) the low-pressure data of Romer ${ }^{14}$ (open circles) and Beal and Hatton $(\mathrm{BH})^{4}$ (full squares) a re shown, while in Fig. $1(b)$ the elevated pressure data of $\mathrm{BH}^{4}$ and recent results by Amend, Eska, and Wiedemann (AEW $)^{15}$ (open circles) are displayed; the data are seen to be in good agreement with theory. We have also included the relaxation time of highly polarized ${ }^{3} \mathrm{He}$ measured by Chapellier, Frossati, and Rasmussen (CFR), ${ }^{16}$ which does not appear to deviate much from the zero-field values of $T_{1}$.

Under usual experimental circumstances there exist further relaxation mechanisms beside the bulk (intrinsic) mechanism studied here, e.g., wall relaxation. However, in all of the abovementioned experiments great efforts were made to eliminate spin relaxation at the wall - at low temperatures this becomes increasingly difficult, of course.

In conclusion, we believe to have demonstrated that the spin-relaxation time in a Fermi liquid provides a probe of the spin dynamics at low to intermediate wave vectors (i.e., on the atomic scale). The polarization-potential approach of Aldrich and Pines to the spin dynamics yields values of the temperature-dependent spin-relaxation time in good agreement with experiment, which may be interpreted as a further successful test of that theory.

Note added.-After submitting the paper, Dr. $\mathrm{H}$. Godfrin kindly informed us that they have recently investigated $^{17}$ the spin-relaxation time $T_{1}$ of liquid ${ }^{3} \mathrm{He}$ at pressures $P=0$ and 22 bars for temperatures $0.05-2 \mathrm{~K}$. No dependence on magnetic field was observed up to $7.3 \mathrm{~T}$. Their data are well described by our theoretical results.

\footnotetext{
${ }^{1}$ For a review, see, e.g., W. G. Stirling, J. Phys. (Paris), Colloq. 39, C6-1134 (1978).

${ }^{2}$ G. H. Aldrich and D. Pines, J. Low Temp. Phys. $\underline{32}$, 689 (1978).

${ }^{3}$ W. Götze and P. Wölfle, J. Low Temp. Phys. $\underline{5}, 575$ (1971).

${ }^{4}$ B. T. Beal and J. Hatton, Phys. Rev. A $\underline{139}, 1751$

(1965) .

${ }^{5}$ K. Bedell and D. Pines, Phys. Rev. Lett. $\underline{45}, 39$ (1980).

${ }^{6} \mathrm{M}$. Pfitzner, private communication.

${ }^{7}$ F. C. Khanna and H. R. Glyde, Can. J. Phys. $\underline{54}, 648$ (1976).

${ }^{8}$ I. P. Ipatova and G. M. Eliashberg, Zh. Eksp. Teor. Fiz. 43, 1795 (1962) [ Sov. Phys. JETP 16, 1269 (1963)].

${ }^{9} \mathrm{~J} . \mathrm{C}$. Wheatley, Rev. Mod. Phys. 47, 415 (1975).

${ }^{10}$ N. Bloembergen, E. M. Purcell, and R. V. Pound, Phys. Rev. 73, 679 (1948).

${ }^{11}$ H. R. Hart, Jr., and J. C. Wheatley, Phys. Rev. Lett. 4, $3(1960)$.

${ }^{12}$ R. L. Garwin and H. A. Reich, Phys. Rev. 115, 1478 (1959).

${ }^{13}$ J. C. Wheatley, Phys. Rev. 165, 30 (1968).

${ }^{14}$ R. H. Romer, Phys. Rev. 117, 1183 (1960).

${ }^{15}$ B. Amend, G. Eska, and W. Wiedemann, unpublished.

${ }^{16}$ M. Chapellier, G. Frossati, and F. B. Rasmussen, Phys. Rev. Lett. 42, 904 (1979).

${ }^{17}$ H. Godfrin, G. Frossati, B. Hebral, and D. Thoulouze, J. Phys. (Paris) 41, C7-275 (1980), and references therein.
} 